\title{
The superluminous transient ASASSN-15Ih as a tidal disruption event from a Kerr black hole
}

\author{
G. Leloudas ${ }^{1,2 \star}$, M. Fraser ${ }^{3}$, N. C. Stone ${ }^{4}$, S. van Velzen ${ }^{5}$, P. G. Jonker ${ }^{6,7}$, I. Arcavi ${ }^{8,9}$, C. Fremling ${ }^{10}$, \\ J. R. Maund"1, S. J. Smartt' ${ }^{12}$, T. Krühler ${ }^{13}$, J. C. A. Miller-Jones ${ }^{14}$, P. M. Vreeswijk1 , A. Gal-Yam',

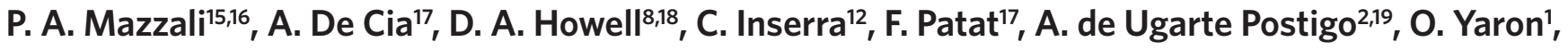 \\ C. Ashall ${ }^{15}$, I. Bar1, H. Campbell3,20, T.-W. Chen ${ }^{13}$, M. Childress ${ }^{21}$, N. Elias-Rosa22, J. Harmanen ${ }^{23}$, \\ G. Hosseinzadeh ${ }^{8,18}$, J. Johansson', T. Kangas ${ }^{23}$, E. Kankare ${ }^{12}$, S. Kim²4 , H. Kuncarayakti²5,26, J. Lyman ${ }^{27}$, \\ M. R. Magee ${ }^{12}$, K. Maguire ${ }^{12}$, D. Malesani' ${ }^{2}$, S. Mattila3,23,28, C. V. McCully ${ }^{8,18}$, M. Nicholl29, S. Prentice ${ }^{15}$, \\ C. Romero-Cañizales ${ }^{24,25}$, S. Schulze ${ }^{24,25}$, K. W. Smith ${ }^{12}$, J. Sollerman ${ }^{10}$, M. Sullivan ${ }^{21}$, B. E. Tucker ${ }^{30,31}$, \\ S. Valenti' ${ }^{32}$, J. C. Wheeler ${ }^{33}$ and D. R. Young ${ }^{12}$
}

When a star passes within the tidal radius of a supermassive black hole, it will be torn apart'. For a star with the mass of the Sun $\left(M_{\odot}\right)$ and a non-spinning black hole with a mass $<10^{8} M_{\odot}$, the tidal radius lies outside the black hole event horizon ${ }^{2}$ and the disruption results in a luminous flare ${ }^{3-6}$. Here we report observations over a period of ten months of a transient, hitherto interpreted ${ }^{7}$ as a superluminous supernova ${ }^{8}$. Our data show that the transient rebrightened substantially in the ultraviolet and that the spectrum went through three different spectroscopic phases without ever becoming nebular. Our observations are more consistent with a tidal disruption event than a superluminous supernova because of the temperature evolution ${ }^{6}$, the presence of highly ionized CNO gas in the line of sight ${ }^{9}$ and our improved localization of the transient in the nucleus of a passive galaxy, where the presence of massive stars is highly unlikely ${ }^{10,11}$. While the supermassive black hole has a mass $>10^{8} M_{\odot}{ }^{12,13}$, a star with the same mass as the Sun could be disrupted outside the event horizon if the black hole were spinning rapidly ${ }^{14}$. The rapid spin and high black hole mass can explain the high luminosity of this event.

ASASSN-15lh was discovered by the All-Sky Automated Survey for Supernovae (ASAS-SN) on 14 June 2015 at a redshift of $z=0.2326$. Its light curve peaked at $V \approx 17$ mag implying an absolute magnitude of $M=-23.5 \mathrm{mag}$, more than twice as luminous as any known supernova ${ }^{7}$. Our long-term spectroscopic follow-up reveals that ASASSN-15lh went through three different spectroscopic phases (Fig. 1). During the first phase ${ }^{7}$, the spectra were dominated by two broad absorption features. While these features appear similar to those observed in superluminous supernovae (SLSNe; Supplementary Fig. 1), their physical origin is different. The features in SLSNe are due to $\mathrm{O}_{\mathrm{II}}^{8,15}$, but this would produce an additional strong feature at $\sim 4,400 \AA$ (Supplementary Fig. 2).

'Department of Particle Physics and Astrophysics, Weizmann Institute of Science, Rehovot 7610001, Israel. 2Dark Cosmology Centre, Niels Bohr Institute, University of Copenhagen, Juliane Maries vej 30, 2100 Copenhagen, Denmark. Institute of Astronomy, University of Cambridge, Madingley Road, Cambridge CB3 OHA, UK. ${ }^{4}$ Columbia Astrophysics Laboratory, Columbia University, New York, New York 10027, USA. ${ }^{5}$ Department of Physics and Astronomy, The Johns Hopkins University, Baltimore, Maryland 21218, USA. ${ }^{6}$ SRON, Netherlands Institute for Space Research, Sorbonnelaan 2, 3584 CA, Utrecht, The Netherlands. ' Department of Astrophysics/IMAPP, Radboud University Nijmegen, PO Box 9010, 6500 GL Nijmegen, The Netherlands. ${ }^{8}$ Las Cumbres Observatory Global Telescope Network, 6740 Cortona Drive Suite 102 Goleta, California 93117, USA. ${ }^{9}$ Kavli Institute for Theoretical Physics, University of California, Santa Barbara, California 93106, USA. ${ }^{10}$ Department of Astronomy, The Oskar Klein Center, Stockholm University, AlbaNova, 10691, Stockholm, Sweden. "Department of Physics and Astronomy, The University of Sheffield, Hicks Building, Hounsfield Road, Sheffield S3 7RH, UK. ${ }^{12}$ Astrophysics Research Centre, School of Mathematics and Physics, Queen's University Belfast, Belfast BT7 1NN, UK. ${ }^{13}$ Max-Planck-Institut für extraterrestrische Physik, Giessenbachstraße, 85748 Garching, Germany. ${ }^{14}$ International Centre for Radio Astronomy Research, Curtin University, GPO Box U1987, Perth, Western Australia 6845, Australia. ${ }^{15}$ Astrophysics Research Institute, Liverpool John Moores University, IC2, Liverpool Science Park, 146 Brownlow Hill, Liverpool L3 5RF, UK. ${ }^{16}$ Max-Planck Institut für Astrophysik, Karl-Schwarzschild-Strasse 1, 85748 Garching, Germany. ${ }^{17}$ European Southern Observatory, Karl-Schwarzschild-Strasse 2, 85748 Garching, Germany. ${ }^{18}$ Department of Physics, University of California Santa Barbara, Santa Barbara, California 93117, USA. ${ }^{19}$ Instituto de Asfrofísica de Andalucía (IAA-CSIC), Glorieta de la Astronomía s/n, E-18008, Granada, Spain. ${ }^{20}$ Department of Physics, University of Surrey, Guildford GU2 7XH, UK. ${ }^{21}$ Department of Physics \& Astronomy, University of Southampton, Southampton SO171BJ, UK. ${ }^{22}$ INAF - Osservatorio Astronomico di Padova, Vicolo dellOsservatorio 5, 35122 Padova, Italy. ${ }^{23}$ Tuorla Observatory, Department of Physics and Astronomy, University of Turku, Väisäläntie 20, FI-21500 Piikkiö, Finland. ${ }^{24}$ Instituto de Asrofísica, Facultad de Física, Pontificia Universidad Católica de Chile, Vicuña Mackenna 4860, 7820436 Macul, Santiago, Chile. ${ }^{25}$ Millennium Institute of Astrophysics, Nuncio Monseñor Sótero Sanz 100 , Providencia, Santiago, 7500011, Chile. ${ }^{26}$ Departamento de Astronomía, Universidad de Chile, Casilla 36-D, Santiago, Chile. ${ }^{27}$ Department of Physics, University of Warwick, Coventry CV4 7AL, UK. ${ }^{28}$ Finnish Centre forAstronomy with ESO (FINCA), University of Turku, Väisäläntie 20, FI-21500 Piikkiö, Finland. ${ }^{29} \mathrm{Harvard}-$ Smithsonian Center for Astrophysics, 60 Garden Street, Cambridge, Massachusetts 02138, USA. ${ }^{30}$ The Research School of Astronomy and Astrophysics, Mount Stromlo Observatory, Australian National University, Canberra Australian Capital Territory 2611, Australia. ${ }^{31}$ ARC Centre of Excellence for All-sky Astrophysics (CAASTRO), Australia. ${ }^{32}$ Department of Physics, University of California, Davis, California 95616, USA. ${ }^{33}$ Department of Astronomy, University of Texas at Austin, Austin, Texas 78712, USA. *email: giorgos@dark-cosmology.dk 


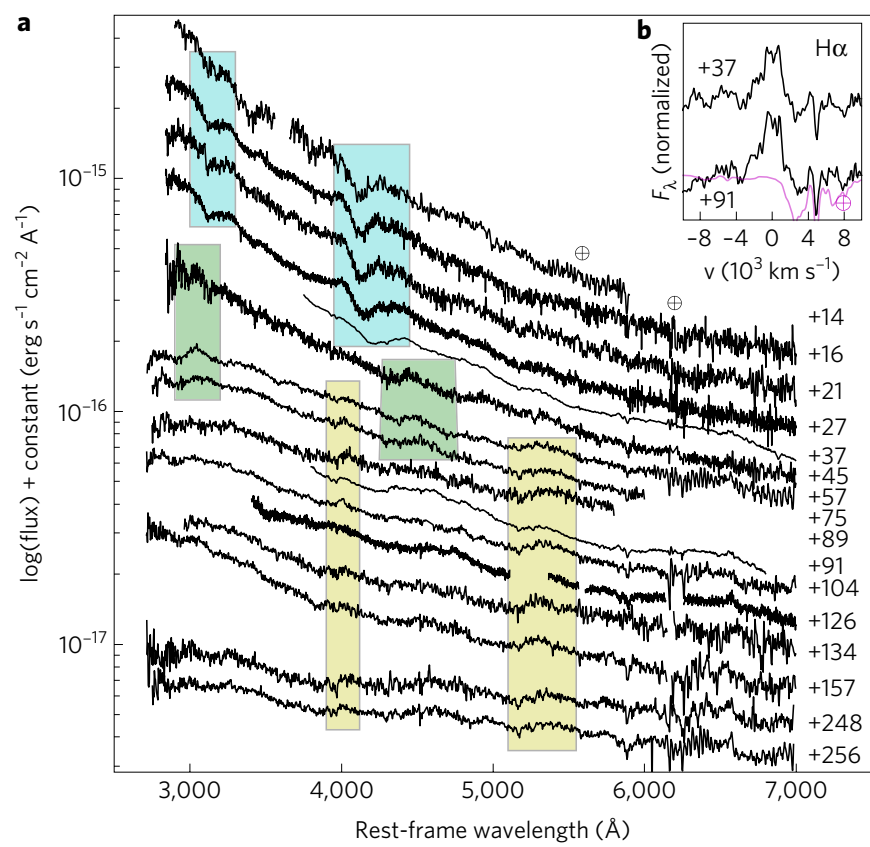

Figure 1 | Spectral sequence of ASASSN-15lh showing three spectroscopic phases. a, The main spectral features during the different phases are highlighted with different colours. The two most recent spectra appear redder due to the increased host contamination. Rest-frame phases are indicated, the spectra have been offset for clarity and the Earth symbols mark the strongest telluric features. b, Detection of $\mathrm{H} \alpha$ (FWHM 2,500 $\mathrm{km} \mathrm{s}^{-1}$ ) in a telluric-free region of our best spectra. The magenta line is a telluric spectrum.

The feature at $\sim 4,100 \AA$ cannot be easily identified in the tidal disruption event (TDE) framework either. Two possibilities are that it could be due to absorption of $\mathrm{Mg}$ II or high-velocity $\mathrm{He}_{\mathrm{II}}{ }^{16}$. After the initial broad absorption features disappeared, the spectra of ASASSN-15lh were dominated by two emission features. A possible identification for these features is He II $\lambda \lambda 3,202$ and 4,686 $\AA$, which are both consistently blue-shifted by $\sim 15,000 \mathrm{~km} \mathrm{~s}^{-1}$ (Supplementary Fig. 3). He II emission is commonly seen in optically discovered TDEs ${ }^{4,5}$ at different blueshifts, albeit typically at lower velocities, but it has not been seen in H-poor SLSNe. These features disappeared after day +75 (measured in rest frame from the peak) and the later spectra were mostly featureless, with the exception of two emission features at $\sim 4,000$ and 5,200 . The spectra remained much bluer than those of $\mathrm{SLSNe}^{17}$ for many months after the peak and never revealed nebular features, even up to day +256 .

A UV spectrum obtained with the HST on day +168 does not show any broad features ${ }^{18}$. At the redshift of the host, we identified weak Ly $\alpha$ absorption and disproportionately strong highionization lines (Nv, Ovi, Civ). Combined with the weakness (or absence) of common ${ }^{19}$ low-ionization absorption lines (FeII, SiII, MgII), this aspect seems to be similar to the spectrum of ASASSN14li, the only available UV spectrum of a $\operatorname{TDE}^{9}$ (see Methods and Supplementary Fig. 4). The highly ionized gas appears at slightly different velocities, suggesting that it could be due to material in the vicinity of the TDE and ionized by its radiation. In contrast to the case of ASASSN-14li', we do not observe any broad features in the UV range, but the optical spectrum is also mostly featureless at these phases.

In addition, we detect the presence of hydrogen in ASASSN-15lh. A weak $\mathrm{H} \alpha$ emission line is unambiguously detected in our highest signal-to-noise spectra (Fig. 1) and in more spectra at lower significance (Supplementary Fig. 3). Its presence cannot be excluded in any spectrum and its strength (equivalent width $\sim 4-8 \AA$ ) is

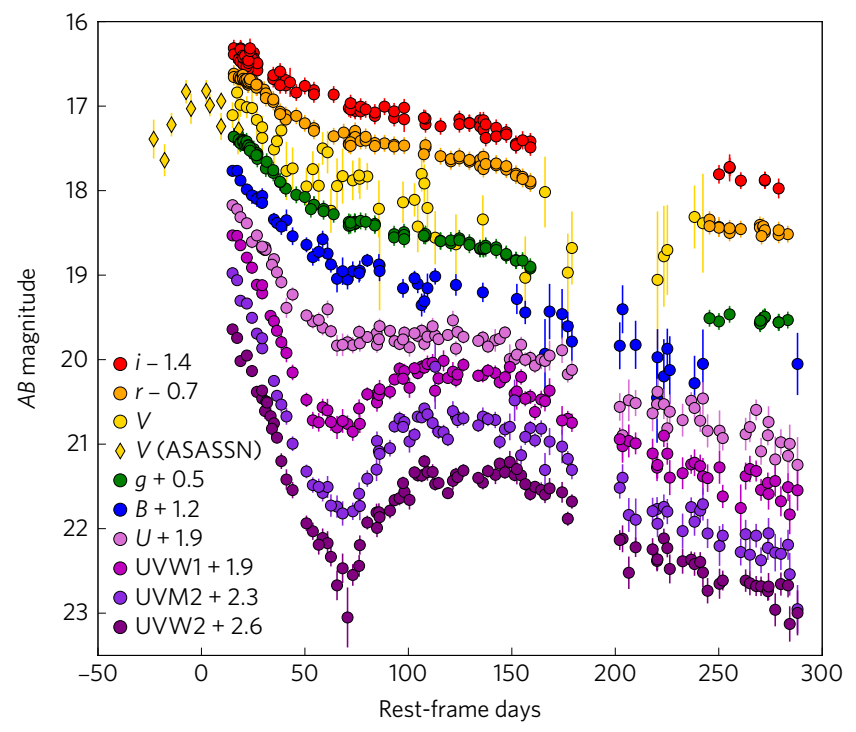

Figure 2 | The light curve evolution of ASASSN-15lh in the rest frame. The data are from LCOGT (gri) and Swift (other filters), supplemented by the ASASSN $V$ band data 7 . We have adopted a peak time at 5 June 2015 (MJD $57178.5)^{7}$. The light curves are shifted for clarity as indicated in the legend. Error bars represent $1 \sigma$ uncertainties. The optical bands show a monotonic decline, but the UV bands show a rebrightening after 60 rest-frame days. A significant secondary dip is also observed in the bluest bands around day +120 . The photometry has been corrected for foreground extinction and the host contribution has been removed (see Methods).

invariable, within the present errors. The velocity of the $\mathrm{H} \alpha$ line (full-width at half-maximum (FWHM) $\sim 2,500 \mathrm{~km} \mathrm{~s}^{-1}$ ) is different from those of other features, implying that it is formed in a different emitting region.

The light curve evolution of ASASSN-15lh is shown in Fig. 2. After the initial peak and decline, around 10 September (day +60), the UV started rebrightening, an effect that was more prominent in the far-UV bands ${ }^{18,20,21}$. The dense photometric follow-up with the Swift Gamma-Ray Burst Mission (Swift) and the Las Cumbres Observatory Global Telescope Network (LCOGT) revealed that ASASSN-15lh reached a secondary UV maximum at around +110 days, followed by another decline. Interestingly, after day +100 , the colours of ASASSN-15lh remained almost constant for over 120 rest-frame days (Supplementary Fig. 5). By fitting a black body to the multiwavelength photometry of ASASSN-15lh, we are able to estimate the temperature evolution, black-body radius and bolometric luminosity (Fig. 3). While the UV region rebrightens and the spectrum changes, the blackbody radius decreases and the temperature increases again, stabilizing at $\sim 16,000 \mathrm{~K}$. This is neither expected from a supernova photosphere, nor observed for SLSNe ${ }^{22-24}$. However, the TDE candidate ASASSN-14ae ${ }^{6}$ showed a very similar temperature evolution to ASASSN-15lh. Even if this happened at shorter timescales, the qualitative similarity between the evolution of the two events suggests that they might be due to the same mechanism. On the other hand, the radius of ASASSN-15lh is larger by a factor of about seven, and ASASSN-14ae has a much stronger $\mathrm{H} \alpha$ line (Supplementary Fig. 1).

By integrating the bolometric luminosity, we estimate that ASASSN-15lh had radiated a total of $1.88 \pm 0.19 \times 10^{52} \mathrm{erg}$ (depending on the assumed bolometric correction) up to 25 May 2016 (day +288). Including kinetic energy, which can reach an additional $10^{52}$ erg for SLSNe ${ }^{15,25}$, the total energy budget approaches the theoretical limit of that which supernova explosion models can accommodate ${ }^{26,27}$. It is possible that UV rebrightening could occur in an SLSN, due to either strong circumstellar interaction or the 


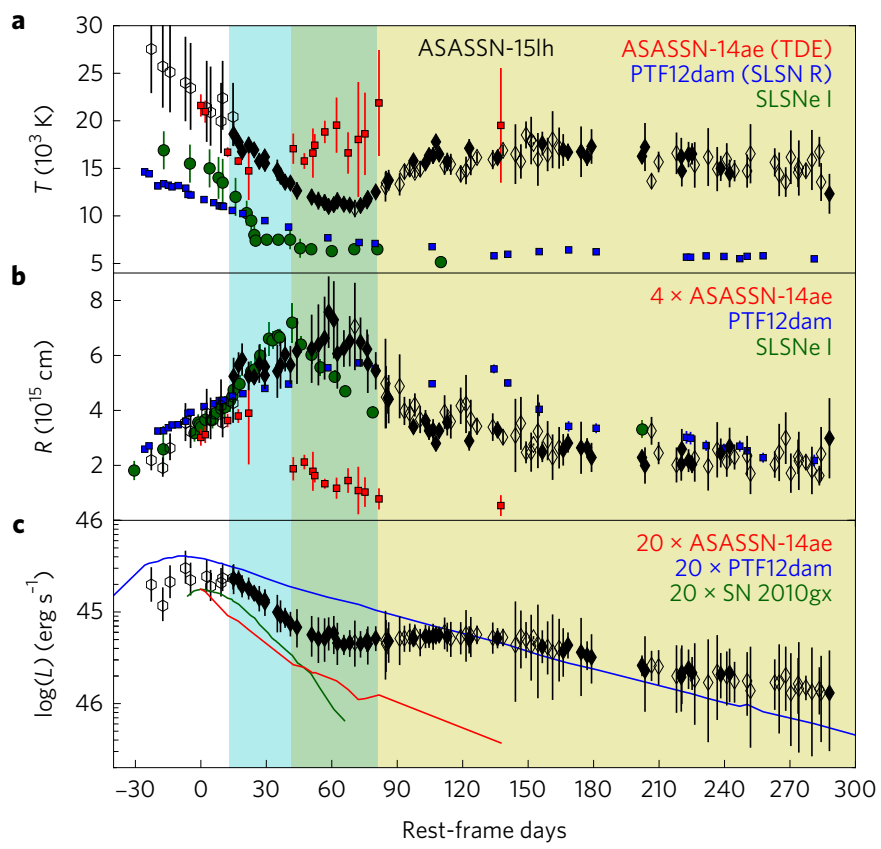

Figure 3 | The evolution of the temperature, radius and luminosity of ASASSN-15Ih compared with TDEs ${ }^{6}$ and SLSNe ${ }^{17,22-24}$. The coloured areas correspond to the different spectroscopic phases in Fig. 1. For ASASSN-15Ih (data in black), open symbols show the fits based on fewer than five filters. In particular, open hexagon symbols show early data, based only on the $V$ band and derived with a temperature prior? Error bars represent $1 \sigma$ uncertainties. The curves are shown with respect to peak time and some comparison objects have been scaled as indicated. $\mathbf{a}, \mathbf{b}$, The evolution of the temperature (a) and radius (b) of ASASSN-15lh are qualitatively similar to those of the TDE ASASSN-14ae, although this is happening on longer timescales and larger radii. All types of SLSNe cool down with time. c, The bolometric luminosity of ASASSN-15lh shows an extended plateau between 70 and 160 days.

ionization breakout powered by a central magnetar. However, the observed $\mathrm{H} \alpha$ line is much weaker than those in SLSNe that have shown signs of late interaction ${ }^{28}$ (Supplementary Fig. 1), and there are no features indicative of interaction in the UV spectrum. In addition, predictions for ionization breakout suggest that the spectrum should turn nebular ${ }^{26}$, although this might apply better to an $\mathrm{X}$-ray rather than a UV breakout. Nevertheless, no detailed model has yet been calculated that can naturally explain the entire spectroscopic and photometric properties observed, either in the SLSN or in the TDE scenario. A single epoch of imaging polarimetry with HST shows low levels of polarization ${ }^{18}$, similar to that obtained for an SLSN $^{29}$ and suggesting an only mildly asymmetric geometry (in projection). Polarization measurements and predictions for optical TDEs are still lacking.

Strong evidence for ASASSN-15lh being a TDE comes from its environment. H-poor SLSNe are found in blue, metal-poor dwarf galaxies with average masses of $\log _{10} M_{\star}=8.24 \pm 0.58 M_{\odot}$, and none have yet been found to exceed $\log _{10} M_{\star}=9.60 M_{\odot}$ at $z<1^{10,11}$. These galaxies typically have strong emission lines, pointing to active ongoing star formation and young progenitor ages that do not exceed a few million years ${ }^{11}$. In contrast, the host of ASASSN-15lh is a massive and passive red galaxy. By fitting the available photometry (see Methods), we estimate that the mass of the host is $\log _{10} M_{\star}=10.95_{-0.11}^{+0.15} M_{\odot}$, with a dominant stellar population of age $3.9_{-1.3}^{+3.2} \mathrm{Gyr}$. The spectral energy distribution (SED) fit provides a star formation rate (SFR) of $0.05_{-0.05}^{+0.15} M_{\odot} \mathrm{yr}^{-1}$, consistent with the improved limit on SFR of

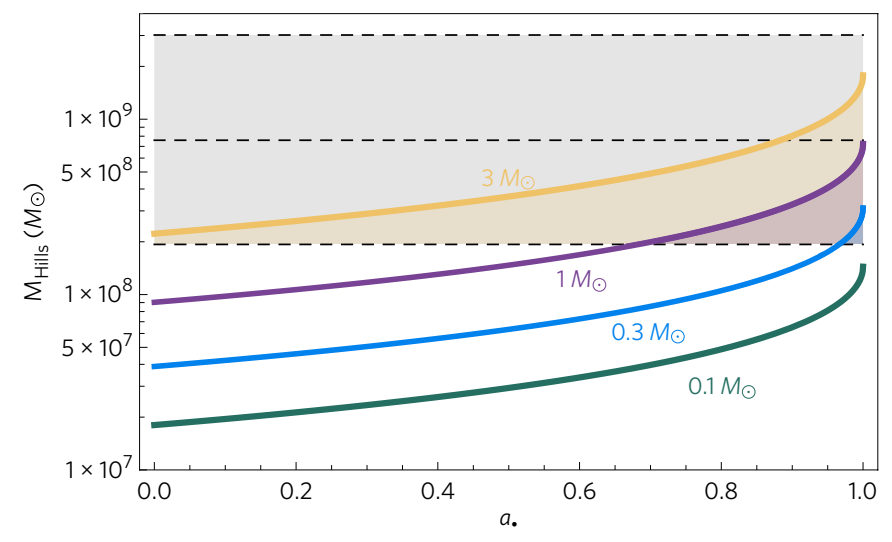

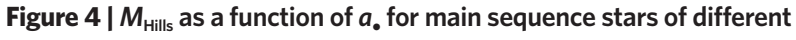
masses. The SMBH mass estimate ${ }^{12}$ for the host of ASASSN-15lh and the corresponding $1 \sigma$ uncertainty region are shown as dashed lines and grey shading. The plot shows that a star of mass $0.1 M_{\odot}$ cannot be disrupted by the $\mathrm{SMBH}$, as the Hills mass is always below the mass of the SMBH. Stars of mass $0.3 M_{\odot}$ and $1 M_{\odot}$ can be disrupted by rotating Kerr black holes of different spins. Only stars of mass $3 M_{\odot}$ lie in the allowed region for TDEs from a non-rotating Schwarzschild black hole, but TDEs from such stars are unlikely ${ }^{30,31}$.

$<0.02 M_{\odot} \mathrm{yr}^{-1}$ that we obtained from our highest signal-to-noise spectrum. The derived specific SFR of $\log \mathrm{sSFR}<-12.5 \mathrm{yr}^{-1}$ is thus three orders of magnitude lower than in any H-poor SLSN host (Supplementary Fig. 7). Furthermore, the transient is positionally coincident with the nucleus of its host. By aligning postdiscovery HST Advanced Camera for Surveys (ACS) images with a prediscovery image taken with the Cerro Tololo Inter-American Observatory $4 \mathrm{~m}$ Dark Energy Camera (Supplementary Fig. 8), we were able to improve the positional accuracy ${ }^{7}$ of ASASSN-15lh by a factor of about four, corresponding to a projected nuclear offset of $131 \pm 192 \mathrm{pc}$.

It has been argued that the large host galaxy mass may imply the presence of a supermassive black hole $(\mathrm{SMBH})$ that is too large to disrupt stars outside its event horizon ${ }^{7}$. Since the tidal radius scales as $R_{\mathrm{t}} \propto M_{\bullet}^{1 / 3}$ while the gravitational radius scales as $R_{\mathrm{g}} \propto M_{\bullet}$, stars can only be disrupted outside the horizon of an SMBH if the black hole is below a certain size, the Hills mass ${ }^{2} M_{\text {Hills }}$. Larger SMBHs swallow stars whole. For a non-spinning Schwarzschild $\mathrm{SMBH}, \quad M_{\mathrm{Hills}} \approx 9 \times 10^{7} M_{\odot} r_{\star}^{3 / 2} m_{\star}^{-1 / 2}$, where $m_{\star} \equiv M_{\star} / M_{\odot}$ and $r_{\star} \equiv R_{\star} / R_{\odot}$ (see Methods). Using an empirical relationship between SMBH mass and total stellar mass for elliptical and spiral/lenticular galaxies with classical bulges ${ }^{12}$, we find $M_{\bullet}$ is far above the Schwarzschild $M_{\text {Hills }}$ for stars of the same mass as the Sun $\log _{10} M_{\bullet}=8.88 \pm 0.60 M_{\odot}$. Using a mass-luminosity relation for early-type galaxies ${ }^{13}\left(M_{\bullet}-L\right.$, using the V-band luminosity), we obtain $\log _{10} M_{\bullet}=8.50 \pm 0.52 M_{\odot}$. However, $M_{\text {Hills }}$ increases by almost an order of magnitude for rapidly spinning Kerr SMBHs and favourable orbital orientations $\mathrm{s}^{14}$. For an optimal (prograde equatorial) orbit and our range of $\mathrm{SMBH}$ mass estimates, we find that a star with the same mass as the Sun can be disrupted by an SMBH with dimensionless spin parameter $a_{\bullet}=0.68$ if $\log _{10} M_{\bullet}=8.28 M_{\odot}$, and by an $\mathrm{SMBH}$ with dimensionless spin parameter $a_{0}=1$ if $\log _{10} M_{\bullet}=8.86 M_{\odot}$. We show the exact relativistic $M_{\mathrm{Hills}}\left(a_{\bullet}\right)$ in Fig. 4 . For stars less massive than the Sun, the spin is constrained to even higher values. ASASSN-15lh could be compatible with a TDE by a Schwarzschild SMBH provided $M_{\star} \approx 3 M_{\odot}$. However, the typical tidally disrupted star comes from the lower end of the stellar mass function, and this hypothesis is further challenged by the old age of the galaxy's stellar population ${ }^{30,31}$. Observations of active galactic nuclei suggest that rapid SMBH spins are common ${ }^{32}$. 
We demonstrated here that TDEs present a method to probe the $\mathrm{SMBH}$ spins of quiescent galaxies. Given the inferred rapid spin of the $\mathrm{SMBH}$, the fact that we did not detect a jet at radio wavelengths implies that black hole spin alone is not sufficient to launch powerful jets (see Methods).

The luminosity and energetics of ASASSN-15lh are also explained by a particularly massive $\mathrm{SMBH}$. The expected radiative efficiency of accretion increases from $\eta \approx 0.05$ (for a Schwarzschild $\mathrm{SMBH}$ ) to $\eta \approx 0.42$ for a rapidly spinning SMBH disrupting stars on prograde, near-equatorial orbits. A particularly massive $\mathrm{SMBH}$ is further biased towards disrupting the most massive stars near the main sequence turn-off mass, increasing accretion rates and total energy release. Finally, it is known that most TDEs only release a small fraction of $\eta M_{\star} c^{2}$ in accretion power (the so-called 'missing energy problem ${ }^{30,33}$ ). One compelling explanation for this is that circularization of debris and formation of the accretion disk is mediated by relativistic apsidal precession, and that the majority of TDEs circularize inefficiently due to weak apsidal precession ${ }^{34}$. For a particularly massive fast-spinning $\mathrm{SMBH}$, efficient circularization is favoured because $R_{\mathrm{t}} \approx R_{\mathrm{g}}$, ensuring large per-orbit precession. The peculiar light curve may also be a natural consequence of tidal disruption by extremely massive black holes. In Methods we combine two competing models (the 'circularization' ${ }^{33}$ and the 'accretion/reprocessing ${ }^{35}$ paradigms) of the optical emission in TDEs and show that the most massive SMBHs produce an unusual hierarchy of tidal disruption timescales. Since the viscous time in the accretion disk is much longer than the debris fallback time when $M_{\bullet}$ is greater than a few $10^{7} M_{\odot}$, TDEs around the most massive SMBHs can display an early peak in the light curve from circularization luminosity, and a second peak from reprocessed accretion luminosity.

\section{Methods}

We describe here the data, methods and theoretical calculations used. We provide details on the localization of ASASSN-15lh and on the host galaxy properties. We present the different data used, and describe the reductions and comment on the implications. Finally, we demonstrate that a TDE can easily accommodate the luminosity and energetics of ASASSN-15lh, we show that combining two luminosity mechanisms for TDEs can result in light curves with two different timescales and we present our derivation of the relativistic $M_{\text {Hills }}$ as a function of the SMBH spin. We assume a Planck cosmology $y^{36}$.

Astrometric localization of ASASSN-15lh. HST observations of ASASSN-15lh were obtained under programme 14348 (PI: Yang) with ACS and a broadband polarimetry filter (POL0V) and the F606W filter. All analyses were performed on the reduced drizzled image obtained from the Barbara A. Mikulski Archive for Space Telescopes (MAST) archive. The image was taken on 3 August 2015, and the total exposure time for this frame was $366 \mathrm{~s}$. The dark energy camera (DECam) image was a $90 \mathrm{~s}$ image taken on 22 October 2014 using an r-band filter. The DECam data were reduced using the Dark Energy Survey Data Management Pipeline and are available online ${ }^{37}$. The measured FWHM was 0.8 arcsec.

Eight sources common to both the DECam and ACS images were used to derive a geometric transformation (allowing rotation, translation and a single scaling factor) between the two frames. Of these sources, five were unresolved in the ACS image and the remaining three had a FWHM of less than about twice that of the point sources. The root mean square error in the transformation was 0.19 DECam pixels, or 52 mas. To measure the position of the host galaxy in the DECam frame, we fitted a model galaxy profile using the GALFIT code; uncertainties in the galaxy centroid were estimated to be only a few hundredths of a pixel from Monte Carlo tests. The position of ASASSN-15lh on the ACS image yielded an accuracy of five hundredths of a pixel (or 3 mas) using three different centring algorithms within the Image Reduction and Analysis Facility PHOT package.

The host galaxy. To derive the physical properties of the host galaxy of ASASSN-15lh through modelling of its SED, we used $\operatorname{griz} Y^{37}, J$ and $K_{\mathrm{s}}^{38}$, as well as $3.4 \mu \mathrm{m}$ and $4.6 \mu \mathrm{m}$ Wide-Field Infrared Survey Explorer photometry. We also performed aperture photometry on Galaxy Evolution Explorer images, yielding no significant detections.

We fitted the galactic extinction-corrected ${ }^{39}$ photometry of the host with stellar population synthesis model ${ }^{40}$ using the photometric analysis for redshift estimate (Le Phare) $\operatorname{code}^{41,42}$. Our galaxy templates were based on a Chabrier initial mass function ${ }^{43}$, and spanned different stellar metallicities, e-folding timescales $\tau$ $(0.1-30 \mathrm{Gyr})$, stellar population ages $(0.01-10 \mathrm{Gyr})$ and dust attenuations ${ }^{44}$. The galaxy stellar mass and SFR are $\log _{10} M_{\star}=10.95_{-0.11}^{+0.15} M_{\odot}$ and $0.05_{-0.05}^{+0.15} M_{\odot} \mathrm{yr}^{-1}$, respectively. Physical parameters are given as the median of the probability distribution of all templates, with error bars containing the $1 \sigma$ probability interval. The best fit model is shown in Supplementary Fig. 6 and it has a low $E(B-V)_{\text {host }}=0.02 \mathrm{mag}$. We assume that the extinction at the host is negligible.

We also constrain the recent star formation in the host by placing limits on the flux of $[\mathrm{O} \mathrm{II}]$ and (narrow) $\mathrm{H} \alpha$. Using the FORS2 spectra (signal-to-noise ratio $>200$ ) we obtain flux limits of $<1.8 \times 10^{-16} \mathrm{erg} \mathrm{s}^{-1} \mathrm{~cm}^{-2}$ for [O II] and $<2.9 \times 10^{-17} \mathrm{erg} \mathrm{s}^{-1} \mathrm{~cm}^{-2}$ for $\mathrm{H} \alpha(2 \sigma)$. These limits constrain the SFR to $<0.25$ and $<0.02 \mathrm{M}_{\odot} \mathrm{yr}^{-1}$ respectively ${ }^{45,43}$, an improvement by a factor of ten over previous estimates?

HST UV spectroscopy. A UV spectrum of ASASSN-15lh was obtained on day +168 with HST under programme 14450 (PI: Brown). We downloaded the reduced Cosmic Origins Spectrograph (COS) and the Space Telescope Imaging Spectrograph (STIS) spectra from the MAST archive. The spectrum does not display any broad emission or absorption features (at similar phases the optical spectrum is also mostly featureless; Fig. 1). We identified both geocoronal and absorption lines at $z=0$ and a number of narrow (FWHM $\sim 200-400 \mathrm{~km} \mathrm{~s}^{-1}$ ) absorption features at the redshift of ASASSN-15lh. Supplementary Fig. 4 shows the COS spectrum (the STIS spectrum is more noisy and less interesting). Supplementary Table 1 contains the equivalent widths and kinematic offsets (measured relative to Ly $\alpha$ ) for lines that were detected, as well as selected limits.

Low ionization features, such as Fe II and Si II, which are common in the star-forming lines of sight of $\mathrm{SLSNe}^{46}$ or gamma ray bursts (GRBs) ${ }^{19,47}$ are weak or absent in ASASSN-15lh. In contrast, absorption from high ionization lines from $\mathrm{N} v$ and $\mathrm{O}$ vi is remarkably strong, especially relative to the (weak) Ly $\alpha$. In particular, the ratio of $\mathrm{N} v$ to Ly $\alpha$ is about four, while it is $>1,000$ times lower in GRBs. The same is true for column densities: using Voigt profile modelling, we derived $\mathrm{N}(\mathrm{H} \mathrm{I})=14.73 \pm 0.12, \mathrm{~N}(\mathrm{O} \mathrm{vI})=15.58 \pm 0.03, \mathrm{~N}(\mathrm{~N} \mathrm{v})=15.42 \pm 0.06$ and $\mathrm{N}(\mathrm{C}$ IV $)=14.60 \pm 0.27$, resulting in ratios that are highly unusual for GRB or quasar damped Lyo systems $\mathrm{s}^{48,49}$, even if the values are lower limits due to saturation. The ratio of $\mathrm{N} v$ to Ly $\alpha$ was also observed to be of the order of unity in the spectrum of ASASSN-14li, the only UV spectrum of a TDE'. By complementing our measurements with those from optical spectra ${ }^{7}$, we found that the Mg II absorption is weak, below the value for GRBs ${ }^{19,47}$ and SLSNe ${ }^{46}$.

Therefore we suggest that the absorbing gas can be separated into two components: (1) a tenuous mildly ionized medium (Ly $\alpha, \mathrm{Mg}$ II, Si III at velocities from 0 to $+44 \mathrm{~km} \mathrm{~s}^{-1}$ ), which is very unusual given the lack of Fe II and $\mathrm{Si}$ II, and (2) a highly ionized medium ( $\mathrm{N} \mathrm{v}, \mathrm{O}$ vi and $\mathrm{C}$ IV) at negative velocity offsets of -80 to $-120 \mathrm{~km} \mathrm{~s}^{-1}$. We suggest that the latter is consistent with material from a disrupted low-mass star, ${ }^{90}$ and that it is ionized by the TDE. Despite the absence of broad features, the phenomenological similarity with the UV spectrum of ASASSN-14li strongly favours a TDE origin for ASASSN-15lh. A highly ionized outflow was also detected for ASASSN-14li in X-rays ${ }^{51}$.

Optical spectroscopy. Spectra were obtained with the instruments and set-ups listed in Supplementary Table 2. The data from FLOYDS, WIFES and EFOSC2 were reduced using dedicated instrument pipelines ${ }^{52-54}$. Spectra from the Very Large Telescope+focal reducer and low dispersion spectrograph (FORS2) and Magellan Telescope+IMACS in the standard fashion using IRAF. The FORS2 spectrum was obtained in spectropolarimetric mode, but the ordinary and extraordinary rays were combined to produce an intensity spectrum.

Imaging and removal of the host contribution. The LCOGT gri images were preprocessed using the Observatory Reduction and Acquisition Control Data Reduction pipeline ${ }^{55}$. To remove the host contribution, we performed image subtraction using the prediscovery DECam gri images as templates. The Swift Ultraviolet and Optical Telescope (UVOT) observations were reduced following the standard procedures and software (uvotsource). To extract the photometry, we used a 4 arcsec aperture and a curve of growth aperture correction. For the Swift filters we did not have prediscovery observations, and hence used the model galaxy spectrum from the SED fit (Supplementary Fig. 6) to generate synthetic magnitudes at these wavelengths. For the B and $\mathrm{V}$ filters, where host contamination is a concern, we estimated the host uncertainty to be $<0.05 \mathrm{mag}$, increasing to $0.1 \mathrm{mag}$ for the $\mathrm{U}$ band. The host uncertainties in the UV filters are more significant, but at these wavelengths the host is many orders of magnitude below the transient luminosity. In the $\mathrm{AB}$ system we obtained host magnitudes of $\mathrm{V}=18.98, \mathrm{~B}=20.43, \mathrm{U}=21.95$, $\mathrm{UVW} 1=23.21, \mathrm{UVM} 2=23.65$ and UVW2 $=23.62 \mathrm{mag}$, which we subtracted from the Swift measurements to obtain the transient photometry. Two UVOT filters suffered from a red leak but this did not affect blue sources such as ASASSN-15lh to the same degree as, for example, $\mathrm{SNe} \mathrm{I}^{56}$. On the basis of synthetic photometry of blackbody spectra, we estimated that the photometry is affected by $<2 \%$ for a black body with $T=15,000 \mathrm{~K}$. As this precision is significantly lower than our photometric accuracy, we did not attempt to correct for this effect. Another study ${ }^{18}$ has found higher values - but still low - for this maximum contamination. 
Radio and X-ray observations. We observed ASASSN-15lh from 05:00 to 14:00 UT on 9 December 2015, using the Australia Telescope Compact Array in configuration 750C, under project code CX340. We observed in two frequency bands of width $2.048 \mathrm{GHz}$ centred at 5.5 and $9.0 \mathrm{GHz}$. We used B1934-638 as both the flux and bandpass calibrator, and B2205-636 as the phase calibrator. We reduced the data following standard procedures in the Multichannel Image Reconstruction, Image Analysis and Display package ${ }^{57}$, and carried out the imaging and self-calibration using the Common Astronomy Software Application ${ }^{58}$.

The field was dominated by PKS J2203-6130, a 9 mJy source (before primary beam correction) located 15 arcmin away from the target. We performed selfcalibration, initially in phase only (down to a timescale of $2 \mathrm{~min}$ ), and eventually in amplitude and phase, on a timescale of $10 \mathrm{~min}$. Given the non-uniform $u v$ coverage, we tested a variety of image weighting schemes, and found the optimum to be a Briggs robust weighting of 0.5. ASASSN-15lh was not detected down to $3 \sigma$ upper limits of 25 and $23 \mu \mathrm{Jy}$ per beam at 5.5 and $9.0 \mathrm{GHz}$, respectively, consistent with a reported upper limit from 3 weeks earlier ${ }^{59}$. Stacking our two frequency bands gave us a slightly deeper $3 \sigma$ radio upper limit of $17 \mu \mathrm{Jy}$ per beam.

The X-Ray Multi-Mirror Mission (XMM-Newton) satellite observed ASASSN 15lh as part of a Director's Discretionary Time proposal on 18 November 2015. The on-source time is $11.9 \mathrm{ks}$, and after filtering epochs of high background, $9 \mathrm{ks}$ of MOS 2 and $4 \mathrm{ks}$ of PN data can be used. Given the lower background and the longer net exposure, we used the MOS2 detector for estimating the upper limit on the source flux. In an aperture of radius 32" centred on ASASSN-15lh we derived a $95 \%$ confidence upper limit ${ }^{60,61}$ of 11 source counts in the $0.15-1 \mathrm{keV}$ band. To convert this to a limit on the flux, we use a temperature of $70 \mathrm{eV}$ found for ASASSN-14li as input, which taking into account that the $M_{\bullet}$ in ASASSN-15lh is close to $8 \times 10^{8} M_{\odot}$, and that of ASASSN-14li is closer to $2 \times 10^{6} M_{\odot}$, implies a black-body temperature of $18 \mathrm{eV}$ (as $T \propto M^{-0.25}$ ). With this and $N_{\mathrm{H}}=3 \times 20 \mathrm{~cm}^{-2}$ W3PIMMS provides a $95 \%$ upper limit to the $0.3-1 \mathrm{keV} \mathrm{X}$-ray flux of $2 \times 10^{-16} \mathrm{erg}$ $\mathrm{cm}^{-2} \mathrm{~s}^{-1}$, yielding an upper limit to the source luminosity of $3 \times 10^{40} \mathrm{erg} \mathrm{s}^{-1}$ This limit depends strongly on the chosen energy band. The $0.3-1 \mathrm{keV}$ band was chosen to allow comparison with ASASSN-14li (ref. 62)

If TDEs do in fact all produce radio jets, as suggested by the recent detection of ASASSN-14li ${ }^{62}$, then applying the same model with appropriate scalings would predict a radio flux of $10 \mu \mathrm{Jy}$ at the time of the observations. The fact that we did not detect a radio jet therefore remains consistent with a TDE origin for ASASSN-15lh, and implies that the jet power of ASASSN-15lh is $\nu L_{\nu} \lesssim 10^{38} \mathrm{erg} \mathrm{s}^{-1}$, which is at least two or three orders of magnitude lower than that of the relativistic TDE Swift J1644 $+57^{63}$. This difference was probably caused by a combination of misalignment of the jet axis and the line of sight (consistent with our non-detection of X-rays) and differences in accretion flow geometry, interstellar medium density or magnetic field strength ${ }^{64}$. If black hole spin were the dominant factor in setting jet power, we would have expected ASASSN-15lh to host a jet at least comparable in strength to that of ASASSN-14li (as we have inferred a high black-hole spin for ASASSN-15lh). Given that our upper limit is close to the ASASSN-14li model prediction, this jet should have been detectable unless the spin of ASASSN-15lh is significantly less than we have inferred, the interstellar medium density is extremely low, or the SMBH in ASASSN-14li is also rapidly spinning.

Tidal disruption luminosities and energetics. In Newtonian theory, a star with mass $M_{\star} \equiv m_{\star} M_{\odot}$ and radius $R_{\star} \equiv r_{\star} R_{\odot}$ will be tidally disrupted when it approaches an SMBH of mass $M_{\bullet}=M_{8} \equiv 10^{8} M_{\odot}$ within a distance:

$$
R_{\mathrm{t}}=R_{\star}\left(\frac{M_{\bullet}}{M_{\star}}\right)^{1 / 3}
$$

Stars passing within this tidal radius can create a luminous electromagnetic flare provided $R_{\mathrm{t}} \gtrsim R_{\mathrm{IBCO}}$, the location of the innermost bound circular orbit (IBCO) for the SMBH. This occurs for SMBHs smaller than the Hills mass,

$$
M_{\mathrm{Hills}} \equiv 9 \times 10^{7} M_{\odot} r_{\star}^{3 / 2} m_{\star}^{-1 / 2}
$$

Once the star has been disrupted, half of its gas is unbound from the $\mathrm{SMBH}$; the other half remains bound with a characteristic spread in specific orbital energy ${ }^{65,66}$

$$
\Delta \epsilon=\frac{G M_{\bullet} R_{\star}}{R_{\mathrm{t}}^{2}}
$$

If we assume a top-hat distribution of debris energy, then the fallback time (in units of seconds) for the most tightly bound debris is:

$$
t_{\mathrm{f}}=3.5 \times 10^{7} M_{8}^{1 / 2} m_{\star}^{-1} r_{\star}^{3 / 2}
$$

which gives a peak fallback rate of:

$$
\frac{\dot{M}_{\mathrm{p}}}{\dot{M}_{\mathrm{Edd}}}=0.13 \eta_{-1} M_{8}^{-3 / 2} m_{\star}^{2} r_{\star}^{-3 / 2}
$$

where $\eta=0.1 \eta_{-1}$ is the radiative efficiency of accretion used to calculate the Eddington-limited mass inflow rate $\dot{M}_{\text {Edd }}$. The peak bolometric luminosity is:

$$
L_{\mathrm{p}}=1.9 \times 10^{45} \eta_{-1} M_{8}^{-1 / 2} m_{\star}^{2} r_{\star}^{-3 / 2} \mathrm{erg} \mathrm{s}^{-1}
$$

which is easily compatible with our observations for $m_{\star}=1$ and $\eta=0.42$, appropriate for Kerr black holes. The radiated bolometric energy is:

$$
E_{\text {rad }}=8.9 \times 10^{52} \eta_{-1} m_{\star} \operatorname{erg}
$$

ASASSN-15lh, although extreme as a supernova ${ }^{26,27,67-69}$, does not strain the theoretical luminosity or energy budgets of TDEs.

Tidal disruption flare timescales. Existing observations do not yet distinguish between two competing theories for producing TDE optical emission. In the accretion/reprocessing paradigm, the bolometric luminosity of a compact $\left(\sim 10 R_{\mathrm{g}}\right)$, efficiently circularized accretion disk is intercepted by an optically thick screen of gas at larger scales $\left(\sim 10^{3} R_{\mathrm{g}}\right)$. This reprocessing layer may be a slow outflow from the $\operatorname{disk}^{70}$, or a more hydrostatic configuration ${ }^{35,71,72}$. However, in both cases it absorbs and re-emits a large fraction of the disk bolometric luminosity at longer wavelengths. Both cases predict a larger reprocessing layer for larger SMBHs: outflow velocities $v_{\mathrm{w}} \sim \sqrt{G M_{\bullet} / R_{\mathrm{t}}} \propto M_{\bullet}^{1 / 3}$, or, if we assume that a hydrostatic reprocessing layer exists at scales comparable to the semimajor axis of the most tightly bound material, its size is proportional to $M_{\bullet}^{2 / 3}$. Alternatively, in the circularization paradigm, observed optical emission arises from shocks between debris streams $s^{33}$, which thermalize and radiate stream kinetic energy at the (generally large) stream self-intersection radius.

The efficiency of circularization (and therefore $\dot{M}_{\mathrm{p}}$ ) depends sensitively on the dimensionless orbital pericentre $R_{\mathrm{p}} / R_{\mathrm{g}}$ : small decreases in $R_{\mathrm{p}}$ quickly move the self-intersection point inwards, increasing the circularization efficiency ${ }^{73,74}$. The low luminosities seen in many optically selected TDEs may reflect the fact that most TDEs have subrelativistic pericentres and circularize inefficiently, so that their peak luminosity is much less than $\eta \dot{M}_{\mathrm{p}} c^{2}$ (ref. 34).

For an extremely massive SMBH with $M_{\bullet} \approx M_{\mathrm{Hills}}$, all TDE pericentres are highly relativistic and circularization is maximally efficient (except possibly if the SMBH spin is rapid and strongly misaligned ${ }^{75,76}$ ). Stream self-intersection points will be $\sim R_{\mathrm{p}}$, near the $\mathrm{IBCO}^{34}$, so the circularization and accretion power will be comparable. It is therefore natural to expect that TDEs from the most massive SMBHs will be brighter and able to attain their theoretical peak luminosity, while smaller SMBHs will often produce TDEs that (due to inefficient circularization) have luminosities well below the peak theoretical luminosity.

We propose that the two peaks in the ASASSN-15lh light curve correspond to two different energy sources: the circularization luminosity, and the accretion luminosity. The former evolves on the fallback timescale $t_{\mathrm{f}}$ while the latter evolves on a viscous timescale,

$$
t_{\mathrm{v}}=\sqrt{\frac{8 R_{\mathrm{p}}^{3}}{G M_{\bullet}}} \alpha^{-1}\left[\frac{H\left(2 R_{\mathrm{p}}\right)}{2 R_{\mathrm{p}}}\right]^{-2}
$$

where we have assumed that the disk outer edge is $2 R_{\mathrm{p}}, \alpha<1$ is the usual ShakuraSunyaev viscosity parameter, and $H / R$ is the disk aspect ratio. For standard TDEs, these two sources of luminosity are difficult to disentangle, because $t_{\mathrm{v}} \ll t_{\mathrm{f}}$ (refs. 77,78): as soon as matter circularizes into a disk, it drains rapidly into the $\mathrm{SMBH}$, and the two sources of luminosity rise and fall together.

However, for very massive SMBHs, $\dot{M}_{\mathrm{p}}<\dot{M}_{\mathrm{Edd}}$, and the accretion disk will be geometrically thin, dramatically increasing $t_{\mathrm{v}}$ and producing an inverted timescale hierarchy: $t_{\mathrm{v}} \gtrsim t_{\mathrm{f}}$. The circularization luminosity can be released promptly, but accretion luminosity is bottlenecked by the long $t_{\mathrm{v}}$. The range of inferred SMBH masses for ASASSN-15lh yield $\dot{M}_{\mathrm{p}} \lesssim \dot{M}_{\text {Edd }}$ (unlike for most other TDE hosts), so it is natural to expect two power sources - circularization and accretion to have two different peaks.

The characteristic decay time of the first peak will be $\sim t_{\mathrm{f}}$, while that of the second will be $t_{\mathrm{v}}$, which is a sensitive function of $\alpha$ and $H / R$. Plausible parameter choices (for example $M_{\bullet}=10^{8} M_{\odot}, \alpha=0.1, H / R=0.03$ ) give a $t_{\mathrm{v}}$ of about 6 months, in agreement with the observed second component of the light curve. However, the first component of the light curve decays on a the timescale of about 1-2 months, unlike the Newtonian estimate of equation (4): $t_{\mathrm{f}} \approx 1 \mathrm{yr}$. In the following subsection, we argue that general relativity effects can increase $\Delta \epsilon$ in TDEs with extremely relativistic pericentres, substantially reducing $t_{\mathrm{f}}$ for this subset of TDEs.

Relativistic alterations to the fallback time. Highly relativistic tidal disruption will alter the Newtonian $\Delta \epsilon$. The frozen-in energy spread $\Delta \epsilon$ can increase by a factor of up to $\sqrt{2}$ (ref. 79), decreasing $t_{\mathrm{f}}$ and increasing $\dot{M}_{\mathrm{p}}$ modestly. However, $\Delta \epsilon$ may increase further by modest spin-orbit misalignments in a TDE for sufficiently relativistic pericentres ${ }^{66}$. Here, we argue that this effect can strongly reduce the fallback time from the Newtonian estimate of equation (4).

The extremity of a TDE is quantified not just by $B \equiv R_{\mathrm{p}} / R_{\mathrm{g}}$, but also by the penetration factor $\beta \equiv R_{\mathrm{t}} / R_{\mathrm{p}}$. TDEs with a large $\beta$ will strongly compress the 
star orthogonal to the orbital plane, causing a vertical collapse with velocity $v_{\mathrm{z}} \approx \beta \sqrt{G M_{\star} / R_{\star}}$. The collapse is reversed near the pericentre, once internal pressure in the disrupted star builds up to the point where it 'bounces' along this vertical axis ${ }^{80}$, receiving an almost impulsive hydrodynamic kick $\sim v_{z}$ along the direction of collapse. The bounce typically has little effect on the energy spread of the debris ${ }^{65,73}$, despite the fact that $V_{\mathrm{p}} v_{\mathrm{z}} \gtrsim \Delta \epsilon$ for large $\beta$ or small $B$ (here $V_{\mathrm{p}}$ is the orbital velocity at the pericentre). One could define a hydrodynamic component of the energy spread, $\Delta \epsilon_{\mathrm{h}} \equiv \mathbf{V}_{\mathrm{p}} \cdot \mathbf{v}_{\mathrm{z}}=V_{\mathrm{p}} v_{\mathrm{z}} \cos \theta$, but in standard TDEs the misalignment angle $\theta=\pi / 2$.

However, for very low $B$, modest spin-orbit misalignment will precess the orbital plane as the star passes through the tidal sphere, partially aligning the axis of vertical collapse with the orbital velocity vector. The per-orbit precession in the line of ascending nodes for a parabolic orbit is ${ }^{81}$ :

$$
\delta \Omega=\sqrt{2} \pi a \cdot\left(\frac{R_{\mathrm{g}}}{R_{\mathrm{p}}}\right)^{3 / 2}
$$

at leading post-Newtonian order. Since most of this shift occurs near the pericentre, we approximate the rotation in the line of ascending nodes between $R_{\mathrm{t}}$ and the bounce (near $R_{\mathrm{p}}$ ) as $\delta \Omega / 2$. The misalignment angle is $\theta\left(\cos \theta \approx \frac{\delta \Omega}{2} \sin I\right.$, where $I$ is the misalignment between spin and orbital angular momentum). Defining a total energy spread $\Delta \epsilon_{\mathrm{tot}} \equiv \Delta \epsilon+\Delta \epsilon_{\mathrm{h}}$, we find (Stone, Kennon, \& Metzger, manuscript in preparation):

$$
\Delta \epsilon_{\mathrm{tot}}=\Delta \epsilon\left(1+a_{\bullet} \beta^{3 / 2} B^{-3 / 2} \sin I\right)
$$

The above arguments are approximate, as the post-Newtonian approximation begins to break down for $R_{\mathrm{p}} \approx R_{\mathrm{g}}$, and we have treated the bounce hydrodynamics impulsively. A detailed examination of this spin-orbit coupling is beyond the scope of this paper, but order unity increases in $\Delta \epsilon$ and decreases in $t_{\mathrm{f}} \propto \Delta \epsilon^{3 / 2}$ are expected for TDEs around SMBHs with $a_{\bullet} \approx 1$ and $B \approx 1$.

Relativistic calculation of Hills mass. Some of the previous Newtonian estimates break down when the star's orbital pericentre $R_{\mathrm{p}} \approx R_{\mathrm{g}}$. In this regime, general relativistic effects are crucial for proper modelling of $M_{\text {Hills }}^{82,14}$. The increased $M_{\text {Hills }}$ arises from two different effects: first, a large $a_{\bullet}$ will lower the IBCO radius significantly, and second, the Kerr tidal field is somewhat stronger than the Schwarzschild equivalent.

To calculate $M_{\text {Hills }}\left(a_{0}\right)$, we employ Fermi normal coordinates to write a local, fully general relativistic tidal tensor ${ }^{83}$. We then employ the accepted formalism ${ }^{14}$ to estimate relativistic $M_{\text {Hills }}$. The results are shown for equatorial orbits (that maximize $M_{\text {Hills }}$ ) in Fig. 4, where we see that the relativistic Kerr $M_{\text {Hills }}$ can be almost an order of magnitude greater than the Newtonian (or Schwarzschild) equivalent.

Data availability. The photometry and spectra of ASASSN-15lh supporting the findings of this study are available from the Weizmann Interactive Supernova Data Repository $^{84}$ (http://wiserep.weizmann.ac.il/).

Received 21 July 2016; accepted 27 September 2016; published 12 December 2016; corrected 22 December 2016

\section{References}

1. Rees, M. J. Tidal disruption of stars by black holes of $10^{6}-10^{8}$ solar masses in nearby galaxies. Nature 333, 523-528 (1988).

2. Hills, J. G. Possible power source of Seyfert galaxies and QSOs. Nature 254, 295-298 (1975).

3. van Velzen, S. et al. Optical discovery of probable stellar tidal disruption flares. Astrophys. J. 741, 73 (2011).

4. Gezari, S. et al. An ultraviolet-optical flare from the tidal disruption of a helium-rich stellar core. Nature 485, 217-220 (2012).

5. Arcavi, I. et al. A continuum of $\mathrm{H}$ - to He-rich tidal disruption candidates with a preference for E+A galaxies. Astrophys. J. 793, 38 (2014).

6. Holoien, T. W.-S. et al. ASASSN-14ae: a tidal disruption event at $200 \mathrm{Mpc}$. Mon. Not. R. Astron. Soc. 445, 3263-3277 (2014).

7. Dong, S. et al. ASASSN-15lh: a highly super-luminous supernova. Science 351, 257-260 (2016).

8. Quimby, R. M. et al. Hydrogen-poor superluminous stellar explosions. Nature 474, 487-489 (2011).

9. Cenko, S. B. et al. An ultraviolet spectrum of the tidal disruption flare ASASSN-14li. Astrophys. J. Lett. 818, L32 (2016).

10. Lunnan, R. et al. Hydrogen-poor superluminous supernovae and longduration gamma-ray bursts have similar host galaxies. Astrophys. J. 787, 138 (2014).

11. Leloudas, G. et al. Spectroscopy of superluminous supernova host galaxies. A preference of hydrogen-poor events for extreme emission line galaxies. Mon. Not. R. Astron. Soc. 449, 917-932 (2015).
12. Reines, A. E. \& Volonteri, M. Relations between central black hole mass and total galaxy stellar mass in the local universe. Astrophys. J. 813, 82 (2015).

13. McConnell, N. J. \& Ma, C.-P. Revisiting the scaling relations of black hole masses and host galaxy properties. Astrophys. J. 764, 184 (2013).

14. Kesden, M. Tidal-disruption rate of stars by spinning supermassive black holes. Phys. Rev. D 85, 024037 (2012).

15. Mazzali, P. A., Sullivan, M., Pian, E., Greiner, J. \& Kann, D. A. Spectrum formation in superluminous supernovae (type I). Mon. Not. R. Astron. Soc. 458, 3455-3465 (2016).

16. Strubbe, L. E. \& Quataert, E. Spectroscopic signatures of the tidal disruption of stars by massive black holes. Mon. Not. R. Astron. Soc. 415, 168-180 (2011).

17. Pastorello, A. et al. Ultra-bright optical transients are linked with type Ic supernovae. Astrophys. J. 724, L16-L21 (2010).

18. Brown, P. J. et al. ASASSN-15lh: a superluminous ultraviolet rebrightening observed by Swift and Hubble. Astrophys. J. 828, 3 (2016).

19. de Ugarte Postigo, A. et al. The distribution of equivalent widths in long GRB afterglow spectra. Astron. Astrophys. 548, A11 (2012).

20. Brown, P. J. Ultraviolet rebrightening of superluminous supernova ASASSN-15lh. Astron. Telegr. 8086 (2015).

21. Godoy-Rivera, D. et al. The unexpected, long-lasting, UV rebrightening of the super-luminous supernova ASASSN-15lh. Preprint at https://arxiv.org/abs/1605.00645 (2016).

22. Inserra, C. et al. Super-luminous type Ic supernovae: catching a magnetar by the tail. Astrophys. J. 770, 128 (2013).

23. Nicholl, M. et al. Superluminous supernovae from PESSTO. Mon. Not. R. Astron. Soc. 444, 2096-2113 (2014).

24. Chen, T.-W. et al. The host galaxy and late-time evolution of the superluminous supernova PTF12dam. Mon. Not. R. Astron. Soc. 452, 1567-1586 (2015).

25. Howell, D. A. et al. Two superluminous supernovae from the early universe discovered by the supernova legacy survey. Astrophys. J. 779, 98 (2013).

26. Metzger, B. D., Margalit, B., Kasen, D. \& Quataert, E. The diversity of transients from magnetar birth in core collapse supernovae. Mon. Not. $R$. Astron. Soc. 454, 3311-3316 (2015).

27. Sukhbold, T. \& Woosley, S. E. The most luminous supernovae. Astrophys. J. Lett. 820, L38 (2016).

28. Yan, L. et al. Detection of broad $\mathrm{H} \alpha$ emission lines in the late-time spectra of a hydrogen-poor superluminous supernova. Astrophys. J. 814, 108 (2015).

29. Leloudas, G. et al. Polarimetry of the superluminous supernova LSQ14mo: no evidence for significant deviations from spherical symmetry. Astrophys. J. Lett. 815, L10 (2015).

30. Stone, N. C. \& Metzger, B. D. Rates of stellar tidal disruption as probes of the supermassive black hole mass function. Mon. Not. R. Astron. Soc. 455, 859-883 (2016).

31. Kochanek, C. S. Tidal disruption event demographics. Mon. Not. R. Astron. Soc. 461, 371-384 (2016)

32. Reynolds, C. S. Measuring black hole spin using X-ray reflection spectroscopy. Space Sci. Rev. 183, 277-294 (2014).

33. Piran, T., Svirski, G., Krolik, J., Cheng, R. M. \& Shiokawa, H. Disk formation versus disk accretion - what powers tidal disruption events? Astrophys. J. 806, 164 (2015).

34. Dai, L., McKinney, J. C. \& Miller, M. C. Soft X-Ray Temperature Tidal Disruption Events from Stars on Deep Plunging Orbits. Astrophys. J. Lett. 812, L39 (2015).

35. Guillochon, J., Manukian, H. \& Ramirez-Ruiz, E. PS1-10jh: the disruption of a main-sequence star of near-solar composition. Astrophys. J. 783, 23 (2014).

36. Planck Collaboration XVI. Planck 2013 results. XVI. Cosmological parameters. Astron. Astrophys. 571, A16 (2014).

37. Melchior, P. et al. Optical broad-band photometry and reference image for APMUKS(BJ) B215839.70-615403.9 / ASASSN-15lh from the Dark Energy Survey. Astron. Telegr. 7843 (2015).

38. Prieto, J. L. et al. APMUKS(BJ) B215839.70-615403.9: The massive host galaxy candidate of ASASSN-15lh. Astron. Telegr. 7776 (2015).

39. Schlafly, E. F. \& Finkbeiner, D. P. Measuring reddening with sloan digital sky survey stellar spectra and recalibrating SFD. Astrophys. J. 737, 103 (2011)

40. Bruzual, G. \& Charlot, S. Stellar population synthesis at the resolution of 2003. Mon. Not. R. Astron. Soc. 344, 1000-1028 (2003).

41. Arnouts, S. et al. Measuring and modelling the redshift evolution of clustering: the Hubble Deep Field North. Mon. Not. R. Astron. Soc. 310, 540-556 (1999).

42. Ilbert, O. et al. Accurate photometric redshifts for the CFHT legacy survey calibrated using the VIMOS VLT deep survey. Astron. Astrophys. 457, 841-856 (2006).

43. Chabrier, G. Galactic stellar and substellar initial mass function. Publ. Astron. Soc. Pacif. 115, 763-795 (2003).

44. Calzetti, D. et al. The dust content and opacity of actively star-forming galaxies. Astrophys. J. 533, 682-695 (2000). 
45. Kennicutt, R. C. Jr Star formation in galaxies along the Hubble sequence. Annu. Rev. Astron. Astrophys. 36, 189-232 (1998).

46. Vreeswijk, P. M. et al. The hydrogen-poor superluminous supernova iPTF 13ajg and its host galaxy in absorption and emission. Astrophys. J. 797, 24 (2014)

47. Christensen, L. et al. A high signal-to-noise ratio composite spectrum of gamma-ray burst afterglows. Astrophys. J. 727, 73 (2011).

48. Fox, A. J., Petitjean, P., Ledoux, C. \& Srianand, R. Hot halos around high redshift protogalaxies. Observations of OvI and $\mathrm{Nv}_{\mathrm{V}}$ absorption in damped Lyman- $\alpha$ systems. Astron. Astrophys. 465, 171-184 (2007).

49. Fox, A. J., Ledoux, C., Vreeswijk, P. M., Smette, A. \& Jaunsen, A. O. High-ion absorption in seven GRB host galaxies at $z=2-4$. Evidence for both circumburst plasma and outflowing interstellar gas. Astron. Astrophys. 491, 189-207 (2008)

50. Kochanek, C. S. Abundance anomalies in tidal disruption events. Mon. Not. R. Astron. Soc. 458, 127-134 (2016).

51. Miller, J. M. et al. Flows of X-ray gas reveal the disruption of a star by a massive black hole. Nature 526, 542-545 (2015).

52. Dopita, M. et al. The Wide Field Spectrograph (WiFeS). Astrophys Space Sci. 310, 255-268 (2007).

53. Dopita, M. et al. The Wide Field Spectrograph (WiFeS): performance and data reduction. Astrophys Space Sci. 327, 245-257 (2010).

54. Smartt, S. J. et al. PESSTO: survey description and products from the first data release by the Public ESO Spectroscopic Survey of Transient Objects. Astron. Astrophys. 579, A40 (2015).

55. Jenness, T. \& Economou, F. ORAC-DR: a generic data reduction pipeline infrastructure. Astron. Comput. 9, 40-48 (2015).

56. Brown, P. J. et al. The absolute magnitudes of type Ia supernovae in the ultraviolet. Astrophys. J. 721, 1608-1626 (2010).

57. Sault, R. J., Teuben, P. J. \& Wright, M. C. H. A retrospective view of MIRIAD. In Astronomical Data Analysis Software and Systems IV Vol. 77 (eds Shaw, R. A., Payne, H. E. \& Hayes, J. J. E.) 433 (Astronomical Society of the Pacific Conference Series, 1995).

58. McMullin, J. P., Waters, B., Schiebel, D., Young, W. \& Golap, K. CASA architecture and applications. In Astronomical Data Analysis Software and Systems XVI Vol. 376 (eds Shaw, R. A., Hill, F. \& Bell, D. J.) 127 (Astronomical Society of the Pacific Conference Series, 2007).

59. Kool, E. C. et al. Radio non-detection of ASASSN-15lh $=$ SN2015L. Astron. Telegr. 8388 (2015).

60. Kraft, R. P., Burrows, D. N. \& Nousek, J. A. Determination of confidence limits for experiments with low numbers of counts. Astrophys. J. 374, 344-355 (1991).

61. Helene, O. Errors in experiments with small numbers of events. Nucl. Instrum. Methods Phys. Res. A 228, 120-128 (1984).

62. van Velzen, S. et al. A radio jet from the optical and $\mathrm{x}$-ray bright stellar tidal disruption flare ASASSN-14li. Science 351, 62-65 (2016).

63. Levan, A. J. et al. An extremely luminous panchromatic outburst from the nucleus of a distant galaxy. Science 333, 199-202 (2011).

64. Tchekhovskoy, A., Metzger, B. D., Giannios, D. \& Kelley, L. Z. Swift J1644+57 gone MAD: the case for dynamically important magnetic flux threading the black hole in a jetted tidal disruption event. Mon. Not. R. Astron. Soc. 437, 2744-2760 (2014)

65. Guillochon, J. \& Ramirez-Ruiz, E. Hydrodynamical simulations to determine the feeding rate of black holes by the tidal disruption of stars: the importance of the impact parameter and stellar structure. Astrophys. J. 767, 25 (2013).

66. Stone, N., Sari, R. \& Loeb, A. Consequences of strong compression in tidal disruption events. Mon. Not. R. Astron. Soc. 435, 1809-1824 (2013).

67. Bersten, M. C., Benvenuto, O. G., Orellana, M. \& Nomoto, K. The unusual super-luminous supernovae SN 2011kl and ASASSN-15lh. Astrophys. J. Lett. 817, L8 (2016)

68. Kozyreva, A., Hirschi, R., Blinnikov, S. \& den Hartogh, J. How much radioactive nickel does ASASSN-15lh require? Mon. Not. R. Astron. Soc. 459, L21-L25 (2016).

69. Chatzopoulos, E. et al. Extreme supernova models for the super-luminous transient ASASSN-15lh. Astrophys. J. 828, 94 (2016).

70. Metzger, B. D. \& Stone, N. C. A bright year for tidal disruptions. Mon. Not R. Astron. Soc. 461, 948-966 (2016).

71. Coughlin, E. R. \& Begelman, M. C. Hyperaccretion during tidal disruption events: weakly bound debris envelopes and jets. Astrophys. J. 781, 82 (2014).

72. Roth, N., Kasen, D., Guillochon, J. \& Ramirez-Ruiz, E. The X-Ray through optical fluxes and line strengths of tidal disruption events. Astrophys. J. 827, 3 (2016).

73. Hayasaki, K., Stone, N. \& Loeb, A. Finite, intense accretion bursts from tidal disruption of stars on bound orbits. Mon. Not. R. Astron. Soc. 434, 909-924 (2013).

74. Shiokawa, H., Krolik, J. H., Cheng, R. M., Piran, T. \& Noble, S. C. General relativistic hydrodynamic simulation of accretion flow from a stellar tidal disruption. Astrophys. J. 804, 85 (2015).

75. Hayasaki, K., Stone, N. \& Loeb, A. Circularization of tidally disrupted stars around spinning supermassive black holes. Mon. Not. R. Astron. Soc. 461, 3760-3780 (2016)
76. Guillochon, J. \& Ramirez-Ruiz, E. A dark year for tidal disruption events. Astrophys. J. 809, 166 (2015).

77. Ulmer, A. Flares from the tidal disruption of stars by massive black holes Astrophys. J. 514, 180-187 (1999).

78. Strubbe, L. E. \& Quataert, E. Optical flares from the tidal disruption of stars by massive black holes. Mon. Not. R. Astron. Soc. 400, 2070-2084 (2009).

79. Kesden, M. Black-hole spin dependence in the light curves of tidal disruption events. Phys. Rev. D 86, 064026 (2012).

80. Carter, B. \& Luminet, J.-P. Tidal compression of a star by a large black hole. I: mechanical evolution and nuclear energy release by proton capture. Astron. Astrophys. 121, 97-113 (1983).

81. Merritt, D., Alexander, T., Mikkola, S. \& Will, C. M. Testing properties of the galactic center black hole using stellar orbits. Phys. Rev. D 81, 062002 (2010).

82. Beloborodov, A. M., Illarionov, A. F., Ivanov, P. B. \& Polnarev, A. G. Angular momentum of a supermassive black hole in a dense star cluster. Mon. Not. R. Astron. Soc. 259, 209-217 (1992).

83. Marck, J.-A. Solution to the equations of parallel transport in Kerr geometry; tidal tensor. Proc. R. Soc. Lond. A 385, 431-438 (1983).

84. Yaron, O. \& Gal-Yam, A. WISeREP: an interactive supernova data repository. Publ. Astron. Soc. Pacif. 124, 668-681 (2012).

\section{Acknowledgements}

We acknowledge support from the European Union FP7 programme through the following European Research Council grants: 320360 (M.F., H.C.), 647208 (P.G.J.), 291222 (S.J.S.), 615929 (M.S.). We also acknowledge: Einstein Postdoctoral Fellowship PF5-160145 (N.C.S.), Hubble Postdoctoral Fellowship HST-HF2-51350 (S.v.V.), STFC grants ST/I001123/1 ST/L000709/1 (S.J.S.) and ST/L000679/1 (M.S.), Australian Research Council Future Fellowship FT140101082 (J.C.A.M.-J.), a Royal Society University Research Fellowship (J.R.M.), a Sofja Kovalevskaja Award to P. Schady (T.Kr., T.-W.C.), a Ramón y Cajal fellowship and the Spanish research project AYA 2014-58381 (A.de U.P.), CONICYT-Chile FONDECYT grants 3130488 (S.K.), 3140534 (S.S.), 3140563 (H.K.), 3150238 (C.R.-C.), a PRIN-INAF 2014 project (N.E.-R.), support from IDA (D.M.), an Ernest Rutherford Fellowship (K.M.), CAASTRO project number CE110001020 (B.E.T.), National Science Foundation grant AST 11-09881 and NASA grant HST-AR-13726.02 (J.C.W.). This work used observation from the Las Cumbres Observatory Global Telescope Network (LCOGT) and was based upon work supported by National Science Foundation grant 1313484. The Australia Telescope Compact Array is part of the Australia Telescope National Facility which is funded by the Australian Government for operation as a National Facility managed by Commonwealth Scientific and Industrial Research Organisation. This work was based partially on observations collected as part of the Public European Southern Observatory Spectroscopic Survey for Transient Objects Survey (PESSTO) under European Southern Observatory (ESO) programmes 188.D-3003 and 191.D-0935, and on observations made with ESO Telescopes at the La Silla Paranal Observatory under programme ID 095.D-0633. We thank M. Della Valle for comments.

\section{Author contributions}

G.L. coordinated the PESSTO observations, was principal investigator (PI) of the FORS2 program, analysed the data and wrote the paper. M.F. provided the astrometric localization and reduced the PESSTO spectra. N.C.S. calculated the relationship between the black hole spin and the Hills mass and edited the manuscript. S.v.V. performed the Swift photometry. P.G.J. analysed the X-Ray Multi-Mirror Mission data and helped coordinate the project. I.A. is the PESSTO PI for TDEs and provided LCOGT data. C.F. made the LCOGT photometry. J.R.M. reduced the FORS2 spectra. S.J.S. is the PI of PESSTO and helped coordinate the project. T.Kr. provided the spectral energy distribution fit of the host galaxy. J.C.A.M.-J. provided the radio observations. P.M.V. helped with the analysis of the spectra. G.L., M.F., N.C.S., S.v.V., P.G.J., I.A., S.J.S., J.C.A.M.-J., A.G.-Y. and P.A.M. contributed to the discussions. A.d.U.P. and A.De C. worked on the UV spectrum. D.A.H. is the PI of the LCOGT observations. C.I. and O.Y. are PESSTO builders and helped with the analysis. F.P., D.M., J.S. and J.C.W. provided FORS2 data and analysis. M.C. and B.E.T. provided the WIFES spectra. S.S. and S.K. provided the Magellan spectrum. G.H., C.V.McC. and S.V. obtained and reduced LCOGT data. E.K., K.M., K.W.S., M.S., and D.R.Y. are PESSTO builders and C.A., J.H., S.M., T.-W.C., T.Ka., S.P., C.R.-C., H.K., M.N., J.L., N.E.-R., H.C., I.B., J.J., M.R.M. contributed PESSTO observations or data reductions. Many authors provided comments on the manuscript.

\section{Additional information}

Supplementary information is available for this paper.

Reprints and permissions information is available at www.nature.com/reprints.

Correspondence and requests for materials should be addressed to G.L.

How to cite this article: Leloudas, G. et al. The superluminous transient ASASSN-15lh as a tidal disruption event from a Kerr black hole. Nat. Astron. 1, 0002 (2016).

\section{Competing interests}

The authors declare no competing financial interests. 


\section{Corrigendum: The superluminous transient ASASSN-15lh as a tidal disruption event from a Kerr black hole}

G. Leloudas, M. Fraser, N. C. Stone, S. van Velzen, P. G. Jonker, I. Arcavi, C. Fremling, J. R. Maund, S. J. Smartt, T. Krìhler, J. C. A. Miller-Jones, P. M. Vreeswijk, A. Gal-Yam, P. A. Mazzali, A. De Cia, D. A. Howell, C. Inserra, F. Patat, A. de Ugarte Postigo, O. Yaron, C. Ashall, I. Bar, H. Campbell, T.-W. Chen, M. Childress, N. Elias-Rosa, J. Harmanen,

G. Hosseinzadeh, J. Johansson, T. Kangas, E. Kankare, S. Kim, H. Kuncarayakti, J. Lyman, M. R. Magee, K. Maguire, D. Malesani, S. Mattila, C. V. McCully, M. Nicholl, S. Prentice, C. Romero-Cañizales, S. Schulze, K. W. Smith, J. Sollerman, M. Sullivan, B. E. Tucker, S. Valenti, J. C. Wheeler and D. R. Young

Nature Astronomy 1, 0002 (2016); published 12 December 2016; corrected 22 December 2016.

In the version of this Letter originally published the estimated energy radiated by ASASSN-15lh up to 25 May 2016 was incorrect and should have read $1.88 \pm 0.19 \times 10^{52} \mathrm{erg}$. 